\title{
Good Neighbor Alternative to Best Response and Machine Learning Based Beamforming and Power Adaptation for MIMO Ad Hoc Networks
}

\author{
Alexandr M. Kuzminskiy, Pei Xiao, Rahim Tafazolli \\ 5G Innovation Center, University of Surrey, UK \\ (a.kuzminskiy,p.xiao,r.tafazolli)@surrey.ac.uk
}

\begin{abstract}
Decentralized joint transmit power and beamforming selection for multiple antenna wireless ad hoc networks operating in a multi-user interference environment is considered. An important feature of the considered environment is that altering the transmit beamforming pattern at some node generally creates more significant changes to interference scenarios for neighboring nodes than variation of the transmit power. Based on this premise, a good neighbor algorithm is formulated in the way that at the sensing node, a new beamformer is selected only if it needs less than the given portion of the transmit power required for the current beamformer. Otherwise, it keeps the current beamformer and achieves the performance target only by means of power adaptation. Equilibrium performance and convergence behavior of the proposed algorithm compared to the best response and regret matching solutions is demonstrated by means of semi-analytic Markov chain performance analysis for small scale and simulations for large scale networks.
\end{abstract}

Keywords- Ad hoc MIMO networks, best response dynamics, regret matching learning, good neighbor rules.

\section{INTRODUCTION}

Multiple-input multiple-output (MIMO) communication techniques have been recognized as one of the main enablers to boost the capacity and spectral efficiency of different wireless communication systems including spectrum sharing ad hoc networks [1]-[3]. Distributed beamforming and power allocation techniques in ad hoc networks can increase system throughput and lower energy consumption. The main limiting factor for such networks is the harsh and generally uncontrollable interference environment, which may compromise user experience leading to a limited applicability of decentralized MIMO technology.

One class of decentralized resource allocation techniques that potentially could be applied to the considered problem is based on the best response dynamics [4], [5] leading to known algorithms such as selfish, greedy, iterative water filling, etc. Existence and convergence to the pure strategy Nash equilibrium (NE) for these algorithms can be established in some special scenarios such as: 1) cooperative resource allocation leading to utility functions that include information on the effect of local actions on interference scenarios for neighboring nodes [5];2) symmetrical interference scenarios, where maximization of the locally estimated utility means utility maximization for coexisting nodes [6]; 3) restricted interference levels for coexisting

We would like to acknowledge the support of the University of Surrey 5GIC (www.surrey.ac.uk/5gic) members for this work. nodes, e.g. as specified in [2], [7]. In the general nonreciprocal non-cooperative scenarios convergence properties cannot be established [4], [5], e.g. because of the "exploitation phenomenon" [4].

One known way to overcome the "exploitation phenomenon" for best response (selfish) algorithms is using machine learning techniques such as regret matching (noregret) [4], [5] that are based on learning history of efficiency of all available actions at all coexisting nodes. Application of the regret matching algorithm for beamforming and power adaptation for MIMO ad hoc networks is addressed in [8]. A general drawback of such solutions is their slow convergence [4]. A potential advantage of hybrid solutions based on interfacing between the best response and machine learning algorithms is pointed out in [4]. One example of such a hybrid algorithm is addressed in [9] in the distributed power control problem. The algorithm exploits switching between the iterative water filling and two machine learning algorithms based on a number of empirical thresholds, which may limit its applicability area.

Another way to improve convergence behavior of selfish resource allocation is introduced in [10] and called as a good neighbor (GN) self-organization. The main idea is that the performance targets should be reached with minimum changes to the current resource allocation. In other words, GN coexisting nodes should not disturb others unless it is really needed. This allows a trade-off between the equilibrium and transient performance leading to controllable convergence behavior without any explicit communications between coexisting subsystems. Application of GN self-organization is addressed in decentralized spectrum sharing in cognitive radio networks [10], [11], and distributed beam scheduling in coexisting multi-RAT networks [12].

In this paper, we apply GN beamforming and power adaptation for MIMO ad hoc networks and compare it with the best response and regret matching solutions in the particular ad hoc network scenario similar to the one in [8] with a limited feedback transmit beamforming and power minimization for the given performance targets. To formulate the GN algorithm for the system under study, we make and justify the critical observation that generally changing the transmit beamforming pattern at some node creates more significant changes to interference scenarios for neighboring nodes than variation of the transmit power. 
This observation allows us to formulate the GN algorithm for beamforming and power adaptation and demonstrate its efficiency compared to the known algorithms by means of semi-analytic Markov chain based performance analysis for small scale and simulations for lager scale networks. Particularly, we use Markov chain modeling to study probabilities of absorption and transitions in different groups of states, which allows us to predict desirable/undesirable convergence behaviour leading to the equilibrium or "voluntary dropout".

The reminder of the paper is organized as follows. Section II describes the system model and problem formulation. Section III justifies our observation regarding different sensitivity of the interference scenarios because of transmit beamformer and power variations and formulates the GN algorithm in the considered scenario. Section IV gives a semi-analytic Markov chain based performance analysis. Section V presents the simulation results. Section VI concludes the paper.

\section{System MODEL AND PROBLEM FORMULATION}

We consider a wireless ad hoc network, which consists of $M$ transmit and receive node pairs equipped with $K$ transmit and $K$ receive antennas sharing the same channel. The transmitted complex symbol stream is $s_{m} \in$ $C$ with $\mathrm{E}\left\{\left|s_{m}\right|^{2}\right\}=1$ for $m=1, \ldots M$. Each node has a receive/transmit beamformer pair $\left(\mathbf{w}_{m}, \mathbf{v}_{n_{m}}\right)$ with $\mathbf{w}_{m}, \mathbf{v}_{n_{m}} \in C^{K}$. Similarly to [8], we assume that the transmit beamforming vector at the $m$ th node $\mathbf{v}_{n_{m}}$ is selected from the Grassmanian codebook $\left[\mathbf{v}_{1} \ldots \mathbf{v}_{N}\right]$ of $N$ unit-norm vectors [13].

The received signal vector $\mathbf{r}_{m} \in C^{K}$ at the $m$ th receiving node is given by

$$
\mathbf{r}_{m}=\sqrt{P_{m}} \mathbf{H}_{m m} \mathbf{v}_{n_{m}} s_{m}+\sum_{l=1, l \neq m}^{M} \sqrt{P_{l}} \mathbf{H}_{m l} \mathbf{v}_{n_{l}} s_{l}+\mathbf{z}_{m}
$$

where $\mathbf{H}_{m l}$ denotes the $K \times K$ MIMO channel between the $l$ th transmitting node and the $m$ th receiving node and is quasi-static, $P_{m} \in\left[P_{\min }, P_{\max }\right]$ is the power of the $m$ th transmitting node, and $\mathbf{z}_{m} \in C^{K}$ is the white Gaussian noise term with zero mean and variance $\sigma^{2}$. The $K \times$ $K$ interference plus noise covariance matrix at the $m$ th receiving node is

$$
\mathbf{R}_{m}=\sum_{l=1, l \neq m}^{M} P_{l} \mathbf{H}_{m l} \mathbf{v}_{n_{l}} \mathbf{v}_{n_{l}}^{*} \mathbf{H}_{m l}^{*}+\sigma^{2} \mathbf{I}_{K}
$$

where $(\cdot)^{*}$ is the conjugate transpose operation and $\mathbf{I}_{K}$ is the $K \times K$ unity matrix. The receive beamforming vector can be formed as

$$
\mathbf{w}_{m}\left(n_{m}\right)=\frac{\mathbf{R}_{m}^{-1} \mathbf{H}_{m m} \mathbf{v}_{n_{m}}}{\mathbf{v}_{n_{m}}^{*} \mathbf{H}_{m m}^{*} \mathbf{R}_{m}^{-1} \mathbf{H}_{m m} \mathbf{v}_{n_{m}}}
$$

and the resulting normalized signal-to-interference plus noise ratio (SINR) at the $m$ th receiving node is

$$
\tilde{G}_{m}\left(n_{m}\right)=\mathbf{v}_{n_{m}}^{*} \mathbf{H}_{m m}^{*} \mathbf{R}_{m}^{-1} \mathbf{H}_{m m} \mathbf{v}_{n_{m}}
$$

leading to the actual SINR $G_{m}\left(n_{m}\right)=P_{m} \tilde{G}_{m}\left(n_{m}\right)$.

We assume that all nodes can precisely estimate their own propagation channels $\mathbf{H}_{m m}$. Also, the receiving nodes can asynchronously sense the interference environment and accurately estimate the interference plus noise covariance matrices $\mathbf{R}_{m}$ corresponding to the transmit beamformers and powers of the neighboring transmitters. The problem is to select the transit beamforming vector at the sensing node $\mathbf{v}_{n_{m}}$, the transmit power $\tilde{P}_{m}$ required to achieve the SINR target $G_{t r g}$

$$
\tilde{P}_{m}=\frac{G_{t r g}}{\mathbf{v}_{n_{m}}^{*} \mathbf{H}_{m m}^{*} \mathbf{R}_{m}^{-1} \mathbf{H}_{m m} \mathbf{v}_{n_{m}}}
$$

and feedback the beamformer index $n_{m}$ and $\tilde{P}_{m}$ to the $m$ th transmitter, which applies beamformer $\mathbf{v}_{n_{m}}$, transmit power $P_{m}=\mathrm{P}\left\{\tilde{P}_{m}\right\}$ and transmits the data stream until the next sensing interval for the $m$ th node, where $\mathrm{P}\{\cdot\}$ denotes projection to the power range $\left[P_{\min }, P_{\max }\right]$.

It is important to note that arbitrary target $G_{t r g}$ may not be achievable for some nodes for the given codebook, power constrains, number of antennas and channel realizations. In this paper we accept a "voluntary dropout" mechanism [14] for all the considered algorithms that deactivates some nodes if they cannot achieve the target SINR for more that predefined $T$ consecutive sensing intervals.

The best response algorithm corresponds to selection of the beamformer that requires the minimum transmit power at each sensing interval

$$
n_{m}^{S L F}=\arg \max _{n=1, \ldots, N} \tilde{G}_{m}(n)
$$

leading to the selfish solution that may suffer from the "exploitation phenomenon" as discussed in Section 1. The regret matching algorithm in [8] gives a learning based solution with its advantages and disadvantages as discussed in Section 1. In Section III, we introduce a GN algorithm in the specified scenario.

\section{GoOd NEIGHBOR BEAMFORMING AND POWER ADAPTATION}

First of all, we show that SINR measured at some sensing node practically does not depend on the power of $M_{0} \leq M$ strongest interference sources for that node if $M_{0}<K$. Let us simplify the notations for the interference-plus-noise covariance matrix and SINR in the case of $M_{0}$ dominant interferences. In this case, a large number of weak interference components can be treated as AWGN. Thus we have

$$
\begin{gathered}
\mathbf{R}=\mathbf{H}_{M_{0}} \tilde{\mathbf{D}} \mathbf{H}_{M_{0}}^{*}+\sigma_{0}^{2} \mathbf{I}_{K}, \\
G=\mu_{0} \mathbf{h}_{0}^{*}\left(\mathbf{H}_{M_{0}} \mathbf{D H}_{M_{0}}^{*}+\mathbf{I}_{K}\right)^{-1} \mathbf{h}_{0},
\end{gathered}
$$

where $\mathbf{h}_{0}$ and $\mathbf{H}_{M_{0}}=\left[\mathbf{h}_{1}, \ldots, \mathbf{h}_{M_{0}}\right]$ are the overall normalized unit norm fast fading propagation channels for the sensing node and the strongest interference sources respectively, $\tilde{P}_{0}$ is the overall signal power for the sensing node depending on the transmit power, path loss and shadowing, $\tilde{\mathbf{D}}=\operatorname{diag}\left(\tilde{P}_{1}, \ldots, \tilde{P}_{M_{0}}\right)$ is the $K \times K$ diagonal matrix 
of the overall power of the interference components, $\sigma_{0}^{2}$ is the overall noise power, $\mu_{0}=\tilde{P}_{0} / \sigma_{0}^{2}$ is the overall received signal to noise ratio, and $\mathbf{D}=\operatorname{diag}\left(\mu_{1}, \ldots, \mu_{M_{0}}\right)$ is the diagonal matrix of the overall interference to noise ratios (INR).

Then, according to the matrix inversion lemma [15], equation (8) can be expressed as

$$
\begin{gathered}
G=\mu_{0} \mathbf{h}_{0}^{*}\left[\mathbf{I}_{K}-\mathbf{H}_{M_{0}}\left(\mathbf{H}_{M_{0}}^{*} \mathbf{H}_{M_{0}}+\mathbf{D}^{-1}\right)^{-1} \mathbf{H}_{M_{0}}^{*}\right] \mathbf{h}_{0} \approx \\
\mu_{0} \mathbf{h}_{0}^{*}\left[\mathbf{I}_{K}-\mathbf{H}_{M_{0}}\left(\mathbf{H}_{M_{0}}^{*} \mathbf{H}_{M_{0}}\right)^{-1} \mathbf{H}_{M_{0}}^{*}\right] \mathbf{h}_{0}
\end{gathered}
$$

if $\mu_{l} \gg 1$ for $l=1, \ldots, M_{0}$ strongest interferences taking into account that matrix $\mathbf{H}_{M_{0}}^{*} \mathbf{H}_{M_{0}}$ in (9) is invertable because of $K>M_{0}$. One can see from (9) that the power of strong interference components practically does not affect SINR at the sensing node.

Another illustration of this property is given in Fig. 1, which shows the simulation results for the input $\mathrm{INR}_{\text {input }}=$ $\mu_{1}=\mu_{2}$ and the INR at the beamformer output

$$
\mathrm{INR}_{\text {output }}=\mathrm{INR}_{\text {input }} \frac{\mathbf{h}_{0}^{*} \mathbf{R}^{-1} \mathbf{H}_{M_{0}} \mathbf{H}_{M_{0}}^{*} \mathbf{R}^{-1} \mathbf{h}_{0}}{\mathbf{h}_{0}^{*} \mathbf{R}^{-2} \mathbf{h}_{0}}
$$

at the receiver of some node, where $\mathbf{h}_{0}, \mathbf{h}_{1}, \ldots, \mathbf{h}_{M_{0}} \sim$ $C N_{K, M_{0}+1}\left(0, \mathbf{I}_{K}\right)$ are independent Gaussian vectors for $K=3, M_{0}=2$.

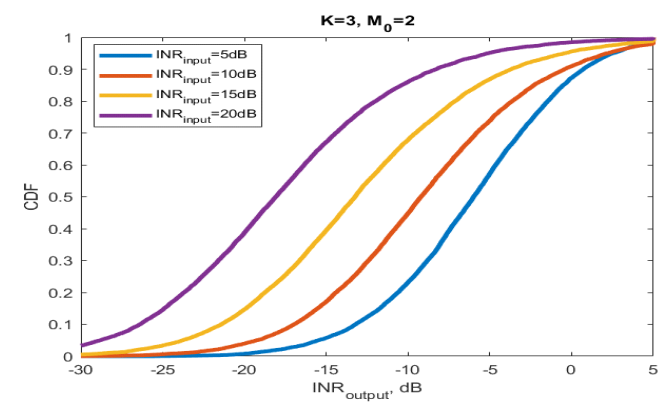

Fig. 1. Illustration of the input/output INR behavior at the receiver

One can see in Fig. 1 that for the relatively high input INR, interference is rejected far below the noise level, therefore if input INR changes, the "current" beamformer remains as efficient as before and does not need to be readjusted. Taking into account that the GN approach is based on minimization of unnecessary changes to the interference environment for the neighboring nodes as the outcome of the actions at the sensing node, we can formulate the GN algorithm in the considered problem as follows:

- At the sensing node, select the new beamformer if it needs less than the given portion of the transmit power required for the current beamformer, otherwise, keep the current beamformer and meet the SINR target only by means of power adaptation.

Now, we can summarize the randomized epsilon greedy GN beamforming and power control algorithm with the following notations: $n_{m}^{(0)}$ is the current beamformer number for the $m$ th sensing node, $0<\alpha \leq 1$ is the GN coefficient, $\epsilon \ll 1$ is the exploration probability and $U$ is the uniform distribution that randomly chooses from 1 to $\mathrm{N}$.
Good neighbor beamforming and power control (GNBPC) algorithm:

1: Data interval

1.1: Transmitter of the $m$ th active node transmits data with transmit power $P_{m}$ using transmit beamformer $\mathbf{v}_{n_{m}}$. 1.2: Receiver of the $m$ th active node receives data with weight vector (3).

2: Sensing interval

2.1: Randomly select the $m$ th sensing node with probability $M^{-1}$.

2.2: Estimate $\mathbf{R}_{m}$ and $\mathbf{H}_{m m}$ (not addressed in the paper, assumed known as indicated in Section II).

2.3: Evaluate $\tilde{G}_{m}(n)$ for $n=1, \ldots, N$ according to (4).

2.4.1: If $\tilde{G}_{m}\left(n_{m}^{(0)}\right) \geq \alpha \max _{n=1, \ldots, N} \tilde{G}_{m}(n)$ and $P_{\text {max }} \tilde{G}_{m}\left(n_{m}^{(0)}\right) \geq G_{t r g}$ then keep $n_{m}=n_{m}^{(0)}$, otherwise go to 2.4 .2 .

2.4.2: Select $n_{m}=\max _{n=1, \ldots, N} \tilde{G}_{m}(n)$ with probability $1-\epsilon$ and $n_{m} \in U$ otherwise.

2.5: Feedback $n_{m}$ and $\tilde{G}_{m}\left(n_{m}\right)$ to the $m$ th transmitter.

2.6: Apply the thransmit beamformer $\mathbf{v}_{n_{m}}$ and update the transmit power $P_{m}=\mathrm{P}\left\{G_{t r g} / \tilde{G}_{m}\left(n_{m}\right)\right\}$.

2.7: Go to 1 .

One can see that GNBPC becomes the epsilon greedy best response (selfish) beamforming and power control algorithm (SBPC) when $\alpha=1$.

\section{SEMI-ANALYtic PERFormance EVAluAtion}

To investigate the convergence and equilibrium properties of the algorithms in Section III, we expand the semi analytic absorbing Markov chain model from [10], [11] to the considered beamforming and power allocation scenario. Semi-analytic (analytic for the given network configuration and propagation channel realizations) Markov chain modeling allows investigation of networks, when convergence to NE (absorbing states in Markov chain terminology) cannot be guaranteed with probability one. Particularly, Markov chain modeling for small scale networks for the given network configuration and propagation channel realizations, allows to find all equilibrium states and ergodic subchains if they exist, enables the analysis of the convergence and non-convergence probabilities and convergence rates. This illustrates performance trends and applicability areas for different algorithms that can be further verified for lager scale networks by means of simulations. Markov chain analysis is used in [10], [11] to study a trade off between equilibrium and transient performance for the GN and selfish algorithms. In the considered problem with the fixed performance targets, we use Markov chain modeling in a different way to study probabilities of absorption and transitions in different groups of states, which allows us to predict desirable/undesirable convergence behaviour leading to the equilibrium or "voluntary dropout".

To formulate Markov chain model of the considered scenario, we introduce the quantized versions of the SBPC and GNBPC algorithms defined in Section III. We assume that only $L$ power levels can be allocated for each node $\mathcal{P}=\left[P_{1}, \ldots, P_{L}\right]$ with $P_{1}=P_{\min }, P_{L}=P_{\max }$. To formulate 
a Markov model we assume that all possible $I=(N L)^{M}$ beamforming and power allocation patterns in the whole network form states of the Markov chain $C_{i}, i=1, \ldots, I$. The current state is fully determined by the current beamforming and power allocations for all nodes. For a given state $C_{i}$, sensing at the $m$ th node transfers the network to states $C_{j_{i m n}}$ with probability

$$
\begin{gathered}
\left\{q_{j_{i m n}}, n=1, \ldots, N\right\}= \\
\left\{\begin{array}{c}
q_{j_{i m n}^{(0)}}=1 ; q_{j_{i m n}}=0, j_{i m n} \neq j_{i m n}^{(0)}, \\
q_{j_{i m n}^{(1)}}=1-\epsilon ; q_{j_{i m n}}=\frac{\epsilon}{N-1}, j_{i m n} \neq j_{i m n}^{(1)},
\end{array}\right.
\end{gathered}
$$

where index $j_{i m n} \in[1, I]$ corresponds to the state number $C_{j_{i m n}}$ with the beamforming and power allocation for transition from the $i$ th state with sensing at the $m$ th node for selection of the $n$th beamformer with the transmit power calculated at Step 2.6 and projected to $\mathcal{P}, C_{j_{i m n}^{(0)}}$ is the state number corresponding to the beamformer selection at $C_{i}$ and power selection at Step 2.6 if the condition at Step 2.4.1 is satisfied, $C_{j_{i m n}^{(1)}}$ is the state number corresponding the beamformer number selected at Step 2.4.2 if the corresponding condition is satisfied with the power defined at Step 2.6.

Assuming that, at each sensing interval, one randomly selected node performs sensing with the uniform probability of $M^{-1}$, the $i$ th row of the transition probability matrix $\mathbf{R}=\left\{r_{i j}\right\}$ can be expressed as

$$
\mathbf{r}_{i}=M^{-1} \sum_{m=1}^{M} \mathbf{r}_{i m}
$$

where $\mathbf{r}_{i m}=\left[0, \ldots,\left\{q_{j_{i m n}}\right\}, \ldots, 0\right]$ is the $1 \times I$ vector with $q_{j_{i m n}}$ probabilities at the $C_{j_{i m n}}$ states.

The transition probability matrix $\mathbf{R}=\left\{r_{j i}\right\}$ is a sparse stochastic matrix with maximum $N M$ non-zero elements in a row such that $\sum_{j=1}^{J} r_{i j}=1, i=1, \ldots, I$, which completely defines the Markov model of the considered spectrum sharing network. Then, for the given scenario realization, algorithm and its parameters, the number of absorbing states (AS) and ergodic subchains (ES), the equilibrium performance, the non-convergence probabilities, and the average number of sensing intervals before absorption can be found similarly to [11] using the states classification algorithm from [16].

A direct investigation of the "voluntary dropout" mechanism with the presented Markov chain model may be difficult. Thus, in this paper, we assume $T=\infty$ and investigate the probabilities to reach ASs: $R_{Q}^{A S}$; states with $P_{m}<P_{\max }$ for $m=1, \ldots M: R_{Q}^{\overline{\max }}$; and states with $P_{m}=P_{\max }$ and $G_{m}<G_{t r g}$ at least for one $m \in[1, \ldots, M]$ : $P_{Q}^{\max }$ after $Q$ sensing intervals. Statistics $P_{Q}^{\max }$ should be related to a possibility to detect situations with $G_{m}<G_{t r g}$ with $P_{m}=P_{\max }$ which may be used as a guidance for selection of the "voluntary dropout" parameter $T$.

Assuming initialization from a uniformly selected states, the $1 \times I$ vector $\mathbf{v}_{Q}$ of probabilities $v_{Q}\left(C_{i}\right)$ to reach the corresponding states of the Markov chain after $Q$ iterations can be expressed as [17]

$$
\mathbf{v}_{Q}=\mathbf{e R}^{Q}
$$

where $\mathbf{e}=\left[I^{-1}, \ldots, I^{-1}\right]$ is the $1 \times I$ initialization vector. Then, the required probabilities can be found as $R_{Q}^{A S}=\sum v_{Q}\left(C_{A S}\right), R_{Q}^{\overline{m a x}}=\sum v_{Q}\left(C_{\overline{\max }}\right)$, and $R_{Q}^{\max }=$ $\sum v_{Q}\left(C_{\max }\right)$, where $C_{A S}$ is the set of absorbing states, $C_{\overline{m a x}}$ and $C_{\max }$ are the corresponding sets of states.

We use the presented semi-analytic approach to evaluate the GNBPC and SBPC performance in the following scenario. We assume that the wireless ad hoc network has $M=3$ node transmitter/receiver pairs randomly located in a $100 \mathrm{~m} \times 100 \mathrm{~m}$ area. A path loss exponent of a radio propagation channel is assumed to be 4 and the noise power $3.16 \cdot 10^{-13} \mathrm{~W}(-95 \mathrm{dBm})$. Each entry in the channel matrix $\mathbf{H}_{m l}$ is assumed to be independent identically distributed complex Gaussian vectors with zero mean and unit variance, which remains unchanged. The target SINR $G_{t r g}$ is selected to be $10 \mathrm{~dB}$ and the power set is $\mathcal{P}=[1,2,5,10,20,50,100] \mathrm{mW}$ for $L=7$. The Grasmanian codebook [13] of $N=4$ elements are used for $K=2$ antennas leading to $I=(7 \cdot 4)^{3}=21952$ states in the considered Markov chain. The exploration probability $\epsilon=0.1$ is used for both SBPC and GNBPC.

The semi-analytic results for 3 typical scenario realizations are presented in Figs. 2-4 for SBPC and GNBPS for $\alpha=[0.75,0.25]$.
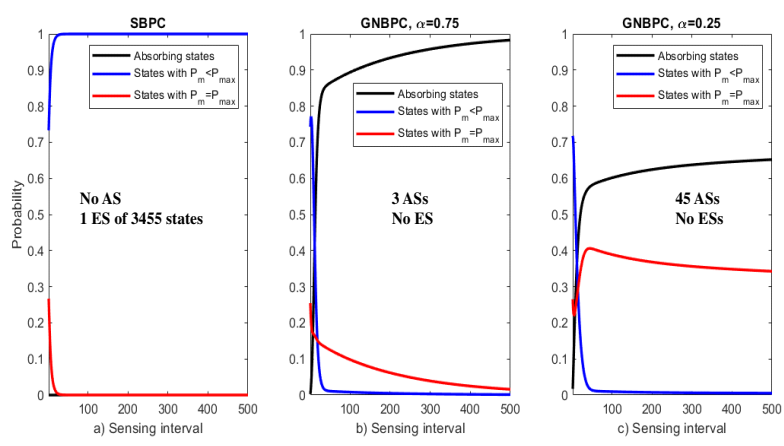

Fig. 2. Scenario realization 1
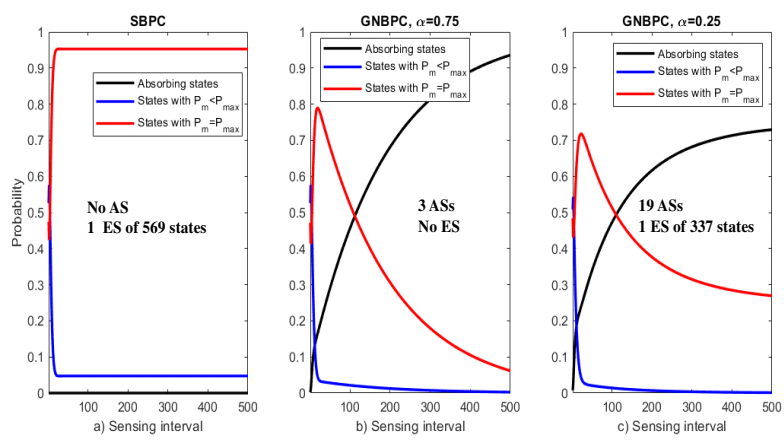

Fig. 3. Scenario realization 2

The following observations can be made from the semianalytic results: 

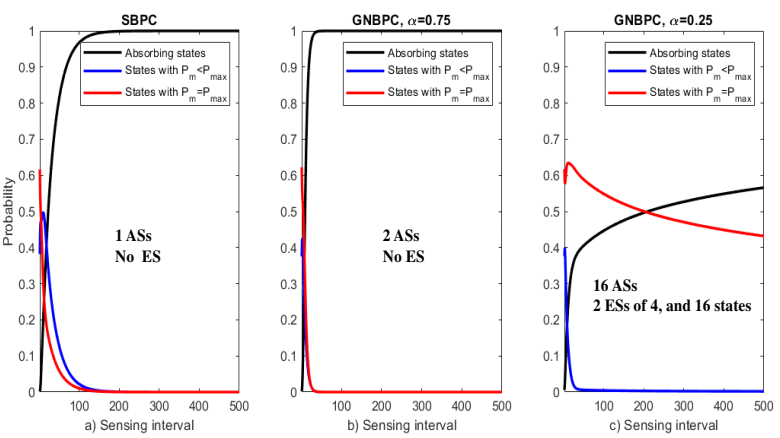

Fig. 4. Scenario realization 3

- The results in Fig. 2 correspond to the difficult scenario for SBPC without any ASs. One can see in Fig. 2a that the chain quickly reaches the ES, which consists mainly of states with $P_{m}<P_{\max }$. This means that the chain never converges and even introduction of a "voluntary dropout" with any parameter $T$ cannot change this situation because $G_{t r g}$ can be reached at vast majority of the ES states. Probably, this situation can be considered as an example of the "exploitation phenomenon" discussed in [4]. GNBPC for $\alpha=0.75$ demonstrates much better convergence behavior in Fig. 2b. Particularly, after 45 sensing intervals, convergence to the ASs is observed with approximately 0.86 probability and transitions mainly with $P_{m}=P_{\max }$ states is observed with approximately 0.13 probability. As a guidance, one could assume that selection of the "voluntary dropout" parameter of $T=45 / M=15$ may activate a "voluntary dropout" of one of nodes with probability close to 0.13 after approximately 45 sensing intervals. The GNBPC convergence behavior for $\alpha=0.25$ in Fig. 2c is much worse, which means that too low values of $\alpha$ should be avoided.

- The results in Fig. 3 correspond to the scenario similar for SBPC, but with the ES consisting mainly of states with $P_{n}=P_{\max }$, which means that impossibility to reach the target performance can be easily detected and a "voluntary dropout" should be practically unavoidable for SBPC for any reasonable $T$. Although the convergence behavior is better for GNBPC as can be seen in Figs. 3b,c, this scenario is difficult also for GNBBC. Particularly, after 150 sensing intervals, one can see practically equal probability for absorption and transitions with $P_{m}=P_{\max }$ states for $\alpha=0.75$.

- The scenario realization in Fig. 4 is simple for both SBPC and GNBPC with approximately twice faster convergence for GNBPC with $\alpha=0.75$ in Fig. 4b compared to the SBPC results in Fig. 4a.

The overall observation is that GNBPC significantly outperforms SBPC in typical scenarios, which needs to be verified for larger scale networks by means of simulations.

\section{NumericAl RESUlts}

We assume a similar to Section IV simulation scenario with $M=12$ node transmitter/receiver pairs separated by maximum $20 \mathrm{~m}$ distance randomly located in a $100 \mathrm{~m}$ $\times 100 \mathrm{~m}$ area. The transmit power is continuous with constraints $P_{\min }=1 \mathrm{~mW}, P_{\max }=100 \mathrm{~mW}$. The Grasmanian codebooks [13] of $N=[8,16]$ elements are used for $K=[3,4]$ antennas correspondingly. The "voluntary dropout" parameter $T$ is selected to be 5 . The same initialization $n_{m}^{(0)} \in U$ is used for all algorithms.

The simulation results in 300 scenario realizations are presented in Fig. 5 for $K=3$ and $N=8$ for SBPC, GNBPC with $\alpha=0.5$, and the regret matching beamforming and power control (RMBPC) machine learning algorithm, which is formulated and applied to the similar scenario in [8]. The results for randomly selected fixed beamforming and power control (FBPC) algorithm are also shown in Fig. 2 for comparison (steps 2.3 and 2.4 above are replaced with $\left.n_{m}=n_{m}^{(0)}\right)$. For all algorithms, the convergence is registered if the transmit beamformers and power remain unchanged during 100 consecutive sensing intervals for the whole network. Particularly, Fig. 2a illustrates the node activation rates for the trials convergent during 3000 sensing intervals for the whole network $(3000 / 12=250$ intervals per node on average). Fig. 2b shows cumulative distribution functions ( $\mathrm{CDF}$ ) for the average transmit power per active node for the convergent trials, and Fig. 2c presents CDFs for the convergence rate for all trials (if some CDF curves end below the unity, then the convergence is not registered for the corresponding portion of the trials for the given algorithm).

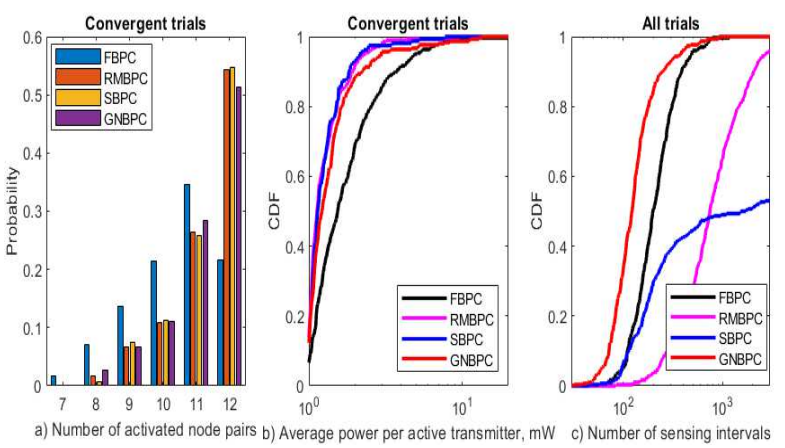

Fig. 5. Simulation results for $K=3, N=8$

The following observations can be made from the simulation results in Fig. 5:

- As expected, FBPC has the lowest activation rate in Fig. 5a, the highest average transmit power in Fig. 5b, but reasonably fast convergence rate of the pure power control.

- All other considered algorithms have a similar activation rate in Fig. 5a.

- SBPC quickly, slowly or never converges to NE depending on scenario realizations as can be seen in Fig. 5c. This is an expected behavior of the best response algorithm, which is explained in [4] by means of an "exploitation phenomenon".

- RMBPC demonstrates much higher probability to reach NE within the considered 3000 sensing intervals compared to SBPC, but its convergence is generally slow as 
shown in Fig. 5c. This behavior is also expected for a learning based technique as discussed in Section I.

- The equilibrium average transmit power performance in Fig. 5b is similar for SBPC and RMBPC and it is the lowest among all considered solutions.

- The proposed GNBPC algorithm demonstrates significant improvement in the convergence rate in Fig. $5 \mathrm{c}$ at the expense of the minor increase of the average transmit power in Fig. $5 \mathrm{~b}$.

One example of convergence behavior for all considered algorithms is shown in Fig. 6 in the case of activation of all 12 nodes in the feasible network configuration for $G_{t r g}=$ $10 \mathrm{~dB}$ ("voluntary dropout" is not used) with the average transmit power per node and the lowest SINR among all nodes in the left and right plots respectively.
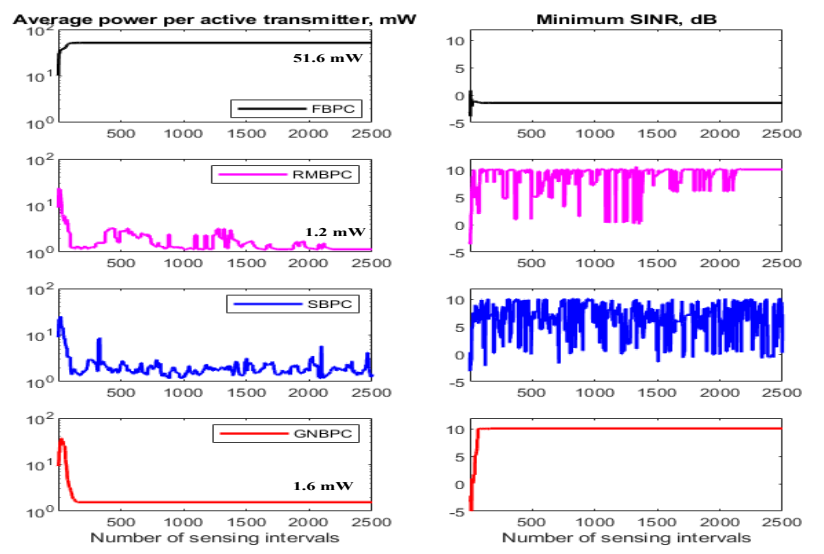

Fig. 6. Example of scenario realization for $K=3, N=8$

The simulation results for $K=4$ and $N=16$ are shown in Fig. 7 in the same scenario and presentation format as in Fig. 5. One can see in Fig. 7:

- Higher selectivity and increased transmit beamforming options lead to significantly higher activation rates for all algorithms in the convergent trials in Fig. 7a compared to Fig. 5a.

- The same factors lead to harsher environment for decentralized non-cooperative beamforming and power adaptation, which in turn results in much slower convergence behavior for both SBPC and RMBPC in Fig. 7c compared to Fig. 5c.

- GNBPC demonstrates even higher convergence rate improvement compared to both SBPC and RMBPC in Fig. 7c than in Fig. 5c at the expense of minor increase in the average transmit power in Fig. $7 \mathrm{~b}$.

\section{Conclusions}

A good neighbor beamforming and power control algorithm has been formulated based on the observation that in the decentralized MIMO ad hoc environment, changing the transmit beamforming pattern at some node generally creates more significant changes to interference scenarios or neighboring nodes than variation of the transmit power. Efficient equilibrium and convergence performance of the

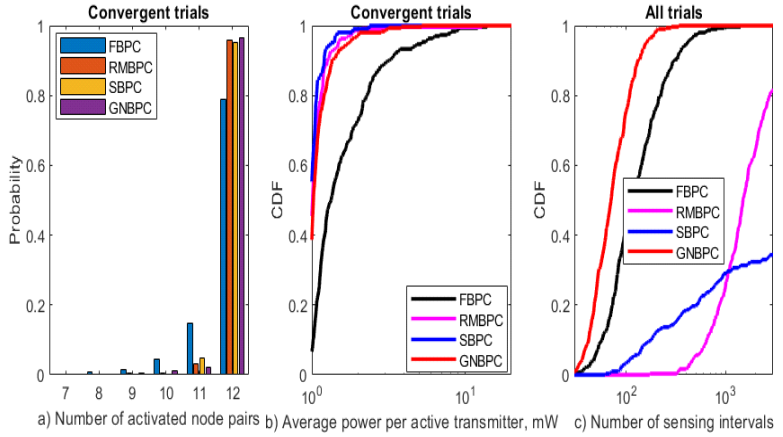

Fig. 7. Simulation results for $K=4, N=16$

proposed algorithm has been demonstrated by means of semi-analytical Markov chain based performance analysis and simulations compared to the best response and regret matching solutions.

\section{REFERENCES}

[1] Spectrum sharing in wireless networks: Fairness, efficiency, and security, J. D. Matyjas, S. Kumar, and F. Hu, Eds. Boca Raton, FL, USA: CRC Press, 2016

[2] D. N. Nguyen, M. Krunz, "Power minimization in MIMO cognitive networks using beamforming games," IEEE J. Selected Areas in Communications, vol. 31, no. 5, pp. 916-925, May 2013.

[3] F. Wilhelmi, C. Cano, G. Neu, B. Bellalta, A. Jonsson, S. Barrachina-Muñoz, "Collaborative spatial reuse in wireless networks via selfish multi-armed bandits," Ad Hoc Networks, 88, pp. 129-141, 2019.

[4] S. Haykin, "Cognitive radio: Brain-empowered wireless communications," IEEE J. Selected Areas in Communications, vol. 23, no. 2, pp. 201-220, Feb. 2005.

[5] N. Nie, C. Comaniciu, "Adaptive channel allocation spectrum etiquette for cognitive radio networks," Mobile Networks and Applications, no. 11, pp. 779-797, 2006.

[6] R. Southwell, J.' Huang, and B. Shou, "Physical interference model based spectrum sharing with generalized spatial congestion games," in Proc. ICCS, pp. 260-264, 2012.

[7] G. Scutari, D. P. Palomar, "MIMO cognitive radio: A game theoretic approach," IEEE Trans. Signal Process., vol. 58, no. 2, pp. 761-779, Feb. 2010.

[8] E. Zeydan, D. Kivanc, U. Tureli, C. Comaniciu, "Joint iterative beamforming and power adaptation for MIMO ad hoc networks," EURASIP J. on Wireless Communications and Networking, vol. 79, no. 1, pp. 1-12, 2011.

[9] O. Ondeng, H. Ouma, "Distributed transmit-power control in cognitive radio networks using a hybrid-adaptive game-theoretic technique," in Proc. AFRICON, Nov. 2015.

[10] A. M. Kuzminskiy, Y. I. Abramovich, "Decentralized dynamic spectrum allocation based on adaptive antenna array interference mitigation diversity," IEEE Trans. on Signal Processing, vol. 58, no.4, pp. 2246-2260, Apr. 2010.

[11] A. M. Kuzminskiy, P. Xiao, R. Tafazolli, "Spectrum sharing with decentralized occupation control in rule regulated networks," IEEE Trans. on Cognitive Communications and Networking, vol. 6, no. 2, pp. 281-294, June 2019.

[12] A. M. Kuzminskiy, P. Xiao, R. Tafazolli, "Good neighbor distributed beam scheduling in coexisting multi-RAT networks," in Proc. WCNC, Apr. 2018.

[13] D. J. Love, R. W. Heath, "Grassmannian beamforming for multiple-input multiple-output wireless systems," IEEE Trans. on Information Theory, vol. 49, no.10, pp. 2735-2747, Oct. 2003.

[14] N. Bambos, "Toward power-sensitive network architectures in wireless communications: Concepts, issues, and design aspects," IEEE Personal Communications, vol. 5, no. 3, pp. 50-59, June 1998.

[15] G. H. Golub, C. F. Van Loan, Matrix computations. Baltimore, MD, USA: Johns Hopkins Univ. Press, 1996.

[16] B. L. Fox, D. M. Landi, "An algorithm for identifying the ergodic subchains and transient states of a stochastic matrix, Commun. ACM, vol. 11, no. 9, pp. 619621, Sep. 1968.

[17] J. G. Kemeny, G. L. Thompson, Introduction to Finite Mathematics. N.J.: Prentice-Hall, 1966. 